\title{
Diseño y validación de la escala de afrontamiento al estrés en padres de hijos con discapacidad
}

\author{
Design and validation of the stress coping scale in parents of children with \\ disabilities
}

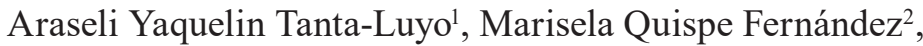 \\ Antonio Serpa Barrientos ${ }^{3}$ \\ Universidad Cesar Vallejo \\ Daniella Elizabeth Ardiles Guevara ${ }^{4}$ \\ Universidad Alas Peruanas
}

Recibido: $02-12-19$

Aceptado: $17-12-19$

\begin{abstract}
Resumen
El objetivo de este artículo consistió en determinar las evidencias psicométricas de la escala de afrontamiento en padres de hijos con discapacidad en Lima. El estudio fue un diseño no experimental, transversal y psicométrico. El muestreo fue no probabilístico y a conveniencia de los investigadores con una muestra de 246 padres de tres centros de educación básica especial, en un rango de edades de 20 a 57 años. El desarrollo de validación se desarrolló con la validez fundamentada en el contenido a través de juicio de expertos y evaluación psicométrica en dos estudios. Los resultados presentaron apropiadas evidencias empíricas de validez por estructura interna, basado en "el modelo unidimensional - Centrado en el problema de 10 ítems (SB$\mathrm{X} 2 / \mathrm{gl}=1.79, \mathrm{CFI}=.92, \mathrm{SRMR}=.06, \mathrm{RMSEA}=.06$ )" $\mathrm{y}$ una consistencia interna, mediante omega compuesto $\omega=.79$. En conclusión, la escala de afrontamiento para padres de hijos con discapacidad evidenció adecuadas propiedades psicométricas, confirmando la dimensión centrado en el problema de la teoría de Lazarus y Folkman (1986).
\end{abstract}

Palabras clave: Discapacidad; afrontamiento; padres.

\section{Abstract}

The objective of this article was to determine the psychometric evidence of the coping scale in parents of children with disabilities in Lima. The study was a non-experimental, cross-sectional and psychometric design. The sampling was not probabilistic and at the convenience

\footnotetext{
1 Universidad César Vallejo. E-mail: yaquelinseli@gmail.com

2 Universidad César Vallejo. E-mail: maquisfer.89@gmail.com

3 Universidad César Vallejo. E-mail: aserpa@ucv.edu.pe,serpa2128@gmail.com

4 Universidad Alas Peruanas. E-mail: daniellardiles@gmail.com

(C) Los autores. Este artículo es publicado por la Revista de Investigación en Psicología de la Facultad de Psicología, Universidad Nacional Mayor de San Marcos. Este es un artículo de acceso abierto, distribuido bajo los términos de la licencia Creative Commons Atribucion - No Comercia_Compartir Igual 4.0 Internacional. (http://creativecommons.org/licenses/by-nc-sa/4.0/) que permite el uso no comercial, distribución y reproducción en cualquier medio, siempre que la obra original sea debidamente citada.
} 
of the researchers with a sample of 246 parents from three special basic education centers, in an age range of 20 to 57 years. The validation development was developed with the validity based on the content through expert judgment and psychometric evaluation in two studies. The results presented appropriate empirical evidence of validity by internal structure, based on "the one-dimensional model - Focused on the problem of 10 items (SB-X2 / gl =1.79, CFI $=.92, \mathrm{SRMR}=.06, \mathrm{RMSEA}=.06)$ " and an internal consistency, using omega compound $\omega=.79$. In conclusion, the coping scale for parents of children with disabilities evidenced adequate psychometric properties, confirming the dimension centered on the problem of the theory of Lazarus and Folkman (1986).

Keywords: Disability; coping; parents.

Al parecer la importancia del afrontamiento al estrés viene siendo investigada en diferentes contextos y en asociación a otras variables, por citar algunas tenemos como: en profesionales médicos (Alosaimi et al., 2018), fuerza armadas (Violanti et al., 2018), adicción al internet en estudiantes universitarios con ideas suicidas (Wei-Po Chou, Cheng-Fang \& Tai-Ling, 2018), mujeres embarazadas con diabetes mellitus (Sürücü, Besen, Duman, \& Erbil 2018), cáncer (Laskowska, 2018), discapacidad intelectual (Barquín-Cuervo, Medina-Gómez \& Pérez de AlbénizGarrote, 2018), Personas trans (Quezada-Berumen, Moral de la Rubia, IbarraGonzález, \& González-Ramírez, 2018), adultos mayores (Herrera, Fernández \& Barros, 2018; Whitehead, \& Bergeman, 2019), parejas que atraviesan tratamiento de fertilidad (Casu, Zaia, Fernandes, Parente \& Gremigni, 2019), cáncer de mama (Lostaunau, Torrejón, \& Cassaretto, 2017), en el desempleo (Díaz, Abal, Climent, Ortega, López-López, \& Casado, 2016) y entre otras. En suma, es diversas y amplias el interés del estudio del estrés a nivel mundial.

Existen diversos autores que conceptualizan el afrontamiento Lazarus y Folkman (1986) mencionaron que "son esfuerzos cognitivos y conductuales constantemente cambiantes" los cuales desarrolla el sujeto debido a las demandas que son evaluadas y percibidas como excedentes de sus recursos. Frydenberg y Lewis (1999) definieron como la capacidad psicosocial del sujeto, donde incluye estrategias conductuales y cognitivas para poder enfrentar situaciones adversas de la vida, mientras que Sandín (2002) señala al afrontamiento como una competencia personal para redirigir las causas y respuesta físicas y psicológicas de los acontecimientos de estrés. Para Folkman \& Moskowitz (2004) el afrontamiento es considerado como un complejo proceso multidimensional que involucra la relación cambiante entre el ambiente, sus demandas y recursos y las disposiciones personales. Por lo tanto, el afrontamiento es visto en muchos casos como un atributo estable de la personalidad y en otros como un proceso altamente situacional (Livneh \& Wilson, 2003); por otra parte, para Connor-Smith y Compas, (2004) el afrontamiento es un proceso transitorio, que se basa en esquemas cognitivos y en la selección de respuestas determinadas ante situaciones específicas. 
Folkman \& Lazarus(1986) en su teoría transaccional del estrés y afrontamiento nos señala que el afrontamiento se divide en dos estilos, el primero centrado en la emoción busca cambiar el sentido de la realidad, asimismo regular o causar una emoción diferente, el segundo centrado en el problema busca modificar la relación entre el entorno y la persona a través de métodos como solución de problemas, toma de decisiones, etc.

Se detectaron diversos agrupamientos de estilos de afrontamiento, donde dos estilos permanecen "emoción" y "problema" (Folkman \& Lazarus ;1986) añade un tercer estilo denominado social (Izquierdo y colaboradores,2008), de igual modo agrega un tercero "evitativo" (Carver, Scheier \& Weintraub, 1989).

El afrontamiento ha sido centro de atención por diversos autores, asimismo empleado en diferentes muestras, reflejado por las múltiples instrumentos existentes, por nombrar algunos de ellos tenemos: Cuestionario de modos de afrontamiento WCQ (Lazarus \& Folkman, 1984; Scherer \& Brodzinski , 1990; Rexrode, 2005; Sawang, Oie, Goh, Mansoer, Markhum \& Ranawake, 2010; Quiroz, Vega \& Soriano, 2010; Rieger, 2016; Charles, Santoro, Edwards, Kang \& Cronan, 2016) versión español (Rodriguez-Marín, Terol, López-Roig \& Pastor,1992), COPE (Carver, Scheier \& Weintraub, 1989; Lyne \& Roger ,2000; Kallasmaa \& Pulver, 2000, Muller \& Spitz,2003; Ingledew, Hardy, Cooper \& Jemal, 2011; Crașovan \& Sava, 2013), adaptado al español (Crespo \& Cruzado, 1997; Cassaretto \& Perez-Aranibar , 2016), COPE Brief (Carver,1997; Perczek, Carver, Price, \& Pozo-Kaderman, 2000; Miyazaki, Bondenhorn, Zalaquett \& KokMun,2008 ;Morán, Landero \& González, 2010; Vargas, Herrera, Rodríguez \& Sepúlveda, 2011; Mate, Andreu \& Peña, 2016; Reich, Costa-Ball \& Remor, 2016 ;Barraza, 2018), Escala de Estrategias de Coping EEC (Chorot y Sandín, 1987 ;Sandín \& Chorot, 2002; Londoño et al., 2006; González \& Landero, 2007), Inventario de Estrategias de Afrontamiento CSI (Tobin, Holroyd \& Reynolds,1982,1983; Tobin, Holroyd, Reynolds, \& Kigal, 1989; Cano, Rodriguez \& Garcia, 2007, Nava, Ollua, Vega \& Soria, 2010; Rodriguez-Diaz, Estrada-Pineda, Rodriguez-Franco, Bringas-Molleda, 2014), Cuestionario de Estilos de Afrontamiento (Roger, Jarvis \& Najarian, 1993; Roger, 1995; Guarino, Sojo \& Bethelmy,2007 ), entre otros.

Según Pozo, Sarria \& Méndez (2006), el estrés familiar ocurre a partir de un estímulo estresor y la baja percepción de la persona de como poder resolverlo, asimismo las familias con hijos con discapacidad están enfocadas en la causa de la discapacidad que en buscar estrategias de solución (Mayo, Taboada, IglesiasSouto \& Dosil, 2012)

Así mismo existen también pruebas que fueron utilizadas para medir el afrontamiento en padres con hijos con discapacidad a nivel internacional: Inventario de Afrontamiento para Situaciones de Estrés (Dabrowska \& Pisula,2010), COPE (Carter, Martínez-Pedraza \& Gray,2009), COPE brief (Hasting et al, 2005; 
Benson,2014; Lai, Goh, Oei \&Sung, 2015), Cuestionario estilo de afrontamiento simplificado (Wang, Hu,Wang, Qin, Xia, Sun \& Wang ,2013; Ji, Zhao, Turner,Sun, Yi \& Tang, 2014) , Cuestionario de modos de afrontamiento (Cappe, Wolf, Bobet, \& Adrien, 2011; Dardas \& Ahmad, 2015), Inventario de Estrategias de Afrontamiento (Luque, Yáñez, Tabernero \& Cuadrado, 2017) y Cuestionario de formas de afrontamiento (Mayo, Real, Toboada, Iglesias-Souto \& Dosil, 2012).

En el Perú, el Instituto nacional de estadística e informática en el Censo Nacional realizado en el año 2017, señaló que 3 millones 51 mil 612 personas de la población peruana sufren de algún tipo de discapacidad $(10,4 \%)$, de la misma manera señala que 692 mil 940 afectados que no cuentan con acceso al seguro de salud (22.7\%), y que 376 mil 891 de estos, no tiene ningún nivel de educación (13,9\%). +

Se encontraron 679 estudios con la palabra afrontamiento, solo uno de ellos es de corte psicométrico (Renati, 2018), manifiestan que existe una escasez de instrumentos para la muestra en mención y la mayoría de las pruebas con ítems en un rango mayor a cincuenta. Padilla, Gómez, Hidalgo y Muñiz (2007) refieren que los resultados de una prueba de cualquier estudio son importantes dado que producen beneficios a la muestra.

Por lo expuesto, el estudio busca diseñar y validar un instrumento que permita identificar las estrategias de afrontamiento en aquellos padres de hijos con discapacidad.

\section{METODO}

\section{Participantes}

La población está conformado por 109095 personas con alguna dificultas o limitación permanente (INEI, 2017). La muestra para esta investigación fue de 246 padres, provenientes de Centros de Educación Básica Especial (CEBES) y las edades de los hijos fueron comprendidos desde los 4 hasta los 17 años. El muestreo fue no probabilístico de tipo intencional, en donde la elección no depende de alguna fórmula, por el contrario, se basó únicamente por la decisión del investigador atendiendo a las características de los participantes (Hernández, Fernández y Baptista, 2014).

En el primer Estudio participaron 54 padres, sus edades fueron comprendidos entre 20 y 57 años, donde $43(83 \%)$ son mujeres y 9 (17\%) hombres. Y, en el segundo estudio participaran un total de 192 padres de familia, de los cuales 141 $(73 \%)$ son mujeres y $51(27 \%)$ varones, con edades de 25 a 55 años.

\section{Instrumentos}

Escala afrontamiento al estrés (EAE) diseñada por los autores, tipo Likert donde: $1=$ casi nunca, $2=$ a veces, $3=$ casi siempre. Lo ítems han sido reflejados 
en actividades que realizarían los padres para afrontar la situación de tener un hijo con discapacidad. El número total de ítems que cuenta la versión final está compuesto por 10 reactivos y con característica unidimensional.

\section{Procedimiento}

\section{Redacción de ítems}

La fase de la creación del instrumento se procedió según los autores (CarreteroDios \& Pérez, 2005), como primer punto se justificó el porqué de un nuevo instrumentos, el segundo se delimitó asumiendo como base la Teoría Transaccional de Lazarus y Folkman (1986)el cuyos planteamientos teóricos del constructo en dos dimensiones; asimismo, artículos publicados en revistas de impacto y orientados en el constructo estrés, discapacidad, familia y afrontamiento (Badia, 2000; Sarriá \& Méndez, 2006; Cabezas, 2001; Fantova, 2000; López, 1999; Parrello \& Caruso, 2007; Pozo, Núñez, 2003). Por último, la construcción y evaluación cuantitativa, se redactó un banco de preguntas para cada dimensión para luego ser revisado por expertos, donde se juzgaron las propiedades de cada ítem (pertinencia, claridad y relevancia). De los cuales 28 ítems obtuvieron resultado de $\mathrm{V}$ Aiken por encima de .70 considerados los más adecuados (Charter, 2003) y, solo dos ítems obtuvieron valores por debajo del umbral y, se optó por eliminarlos.

\section{Recojo de información en campo}

Se continuo la investigación solicitando las respectivas autorizaciones a los Centros Educativos de Básica Especial para la aplicación del instrumento de 28 ítems (fase piloto); posterior a ello, los directores otorgaron los permisos para realizar las evaluaciones a los padres de familia, donde se explicó que la participación es de carácter voluntaria. De esta manera se cumple con el consentimiento informado.

Posterior a ello, fue realizado el primer análisis estadístico de los ítems en una muestra piloto conformado por 54 participantes, con la finalidad de verificar el comportamiento de los reactivos como: asimetría, curtosis y relación ítem total corregido. Una vez analizado los resultados del primer estudio piloto, se procedió a realizar adecuación necesaria a los reactivos y así como también la eliminación de algunos ítems que no cumplieron los criterios antes descritos; para, luego ser nuevamente aplicados a una muestra mayor (segundo estudio piloto) con la finalidad de obtener mejores ajustes de la estructura final de la escala en construcción.

\section{Proceso de análisis de los datos}

El análisis de la normalidad univariada se estimó a través de la asimetría y curtosis en los valores establecidos de -1.5 a 1.5 (Bandalos \& Finney, 2010; Muthén \& 
Kaplan, 1985). En cuanto a la obtención de la normalidad multivariado se evaluó mediante el coeficiente de Mardia obteniendo valor menor a 70 (Mardia, 1970,1974).

En cuanto se refiere al análisis factorial exploratoria, se empleó el programa con acceso libre, como es el FACTOR 10.3 (Lorenzo-Seva \& Ferrando, 2015), en donde los valores de la estimación de un adecuado muestreo a través del KMO y esfericidad de Barlett justificaron la realización de esta técnica multivariado. La obtención de los factores fue a través del método de mínimos cuadrados diagonalizados, por su parte el método de rotación empleado fue Promin debido a que los factores son oblicuos (Lorenza-Seva,1999). La agrupación de estos reactivos a un determinado factor fue asumida con pesos factoriales $\geq .30$ (Kline, 2010).

Con respecto a la evidencia de validez de la estructura interna del constructos, se realizó a través del análisis factorial confirmatorio con el programa R Studio 3.0.1 bajo los siguientes criterios: se utilizó los índices de ajuste convenientemente a la distribución normal y recomendada (Hooper, Coughlan, \& Mullen, 2008): la estimación de SB- $X^{2}$ (Satorra \& Bentler, 2001), la proporción entre SB- $X^{2} / \mathrm{gl}$, los valores estimados por debajo o iguales a 2 se considera un buen ajuste (Gana \& Broc, 2019; Beaducel \& Herzberg, 2006; Tabachnick \& Fidell, 2007); con valores de índice de bondad de ajuste $\geq .90$ (Marsh \& Tai Hau, 1996); sin embargo, los criterios según Hu \& Bentler (1999) consisten en que el CFI, TLI se debe de interpretar valores $\geq .90 \mathrm{y}$, el SRMR y RMSEA $\leq .05$. Por último, el análisis de la confiabilidad se estimó en relación del método de consistencia interna, empleando el coeficiente omega con valores $\geq .70$, considerados como adecuados.

\section{RESULTADOS}

\section{Estudio 1}

Se verifico los descriptivos de cada uno de los ítems (Tabla 1), los resultados permitieron identificar que 13 ítems $(3,6,7,8,11,13,14,15,21,22,26,27$ y 28) no cumplieron con el índice de homogeneidad (ritc: inferiores a .30), los cuales se eliminaron debido a que las correlaciones se encontraron por debajo de lo establecido (Muñiz, 2005). Asi mismo, la asimetría y curtosis se encontraron dentro del rango esperado [-1.5, 1.5] (George \& Mallery, 2003). Las medidas de adecuación muestral fueron óptimas $(\mathrm{KMO}=.75$, Bartlett $p<.000)$.

Con respecto a la estructura factorial exploratoria se observa en la tabla 2, el F1 (Centrado en el problema) está compuesto por ocho ítems y con cargas factoriales comprendidos entre .48 a .90 y el F2 (Centrado en la emoción) conformado por ocho reactivos con pesos factoriales desde .30 a .96. Al revisar las comunalidades se observaron que los ítems son mayores a .30, siendo adecuados (Kline, 2010). En los resultados de consistencia interna según el 
coeficiente de Alfa y Omega reportando para el F1 $(\alpha=.83, \omega=.85)$ y F2 $(\alpha=.80$, $\omega=.80$ ), resultados como aceptables (Campos-Arias \& Oviedo, 2008) y con varianza total explicada de $42.5 \%$.

Tabla 1

Análisis de los puntuaciones extremas 1 (con 28 items y 54 participantes)

\begin{tabular}{|c|c|c|c|c|c|}
\hline Ítems & $\mathrm{M}$ & DS & g1 & g2 & ritc \\
\hline Item1 & 2.72 & 0.45 & -1.02 & -1.00 & .230 \\
\hline Item2 & 2.61 & 0.63 & -1.39 & 0.87 & .490 \\
\hline Item3* & 1.93 & 0.84 & 0.14 & -1.59 & -.030 \\
\hline Item4 & 2.63 & 0.52 & -0.96 & -0.24 & .230 \\
\hline Item5 & 2.65 & 0.59 & -1.48 & 1.25 & .210 \\
\hline Item6* & 2.15 & 0.86 & -0.29 & -1.58 & .090 \\
\hline Item7* & 2.31 & 0.75 & -0.31 & -0.66 & .020 \\
\hline Item8* & 2.63 & 0.62 & -1.49 & 1.14 & .190 \\
\hline temI9 & 2.69 & 0.51 & -1.26 & 0.56 & .470 \\
\hline Item10 & 2.57 & 0.63 & -1.22 & 0.43 & .280 \\
\hline Item11* & 1.72 & 0.71 & 0.46 & -0.89 & .150 \\
\hline Item12 & 2.44 & 0.57 & -0.40 & -0.77 & .381 \\
\hline Item13* & 2.06 & 0.76 & -0.09 & -1.25 & .192 \\
\hline Item14* & 2.50 & 0.82 & -1.18 & -0.43 & .040 \\
\hline Item15* & 2.09 & 0.71 & -.13 & -0.94 & .090 \\
\hline Item16 & 1.96 & 0.70 & 0.05 & -0.89 & .421 \\
\hline Item17 & 2.54 & 0.75 & -1.27 & 0.05 & .590 \\
\hline Item 18 & 2.57 & 0.54 & -0.69 & -0.75 & .270 \\
\hline Item19 & 2.33 & 0.75 & -0.64 & -0.94 & .340 \\
\hline Item 20 & 2.04 & 0.75 & -0.06 & -1.19 & .421 \\
\hline Item $21^{*}$ & 2.13 & 0.58 & -0.02 & -0.05 & -.131 \\
\hline Item $22^{*}$ & 2.44 & 0.74 & -0.94 & -0.53 & -.051 \\
\hline Item 23 & 2.20 & 0.76 & -0.37 & -1.17 & .282 \\
\hline Item 24 & 2.37 & 0.71 & -0.68 & 0.72 & .422 \\
\hline Item 25 & 2.59 & 0.53 & -0.77 & -0.60 & .382 \\
\hline Item $26^{*}$ & 2.07 & 0.87 & -0.15 & -1.67 & .150 \\
\hline Item $27^{*}$ & 1.81 & 0.68 & 0.24 & -0.76 & -.071 \\
\hline Item $28^{*}$ & 1.65 & 0.62 & 0.40 & -0.61 & -.152 \\
\hline
\end{tabular}

Nota $: \mathrm{M}=$ Media; $\mathrm{DS}$ = Desviación Estándar; g1 = Asimetría; $\mathrm{g} 2$ = Curtosis; ritc= ítem total corregido;

$*=$ ítem $<20$ 
Tabla 2

Análisis factorial exploratorio de la Escala de Afrontamiento, estudio $1(\mathrm{n}=54)$

\begin{tabular}{lll}
\hline Ítems & F1 & F2 \\
\hline Ítem1 & .570 & \\
Ítem2 & .640 & \\
Ítem4 & .770 & \\
Ítem5 & .570 & \\
Ítem9 & .490 & \\
Ítem10 & .700 & \\
Ítem12 & .910 & .440 \\
Ítem16 & .610 & .400 \\
Ítem17 & & .310 \\
Ítem18 & & .630 \\
Ítem19 & & .740 \\
Ítem20 & & .960 \\
Ítem23 & & .530 \\
Ítem24 & & .616 \\
Ítem25 & & - \\
\hline F1 & & \\
F2 & & \\
\%varianza total & & $42.5 \%$ \\
\hline
\end{tabular}

Nota. F1: Centrado en el problema, F2: centrado en la emoción

Estudio 2

Descripción de puntajes extremos

De acuerdo a los resultados obtenidos del análisis descriptivo (tabla 3), Escala de afrontamiento al estrés presentaron valores de asimetría y curtosis dentro del rango esperado $[-1.5,1.5]$. Con respecto al cumplimiento del supuesto de normalidad multivariada no se obtuvo un coeficiente de Mardia $<5.00$ (G2=12.29, $\mathrm{Z}=-3.256$, $\mathrm{p}<.00$; Mardia, 1970).

\section{Estructura interna del instrumento}

De acuerdo al análisis factorial confirmatorio, se obtuvo tres modelos (véase la Tabla 4), en donde el modelo 1 con 14 ítems, excepto el ítem 13, se observa valores por debajo del umbral establecido en el AFE, el modelo 2 con 13 ítems eliminando, el ítem 23 por mostrar peso factorial por debajo del umbral mínimo establecido $(\lambda<.30)$ en el AFC y el modelo 3 unifactorial (Centrado en el problema) con 10 ítems). Asimismo, Se calculó la consistencia interna a través de índice omega de McDonald (1999) para los modelos 1,2 y 3, donde el Factor 1 de los tres modelos 
propuestos se obtuvo $\omega=.79$ y el Factor 2 del Modelo 1 se obtuvo $\omega=.37$ y Modelo $2 \omega=33$. En suma, la consistencia interna para los tres modelos del primer factor se han obtenido valores aceptables; sin embargo, para el segundo factor F2 de los dos modelos (Modelo 1 y Modelo 2) la consistencia interna no son los más adecuados, por lo tanto, se procedió retirar el F2, logrando índices de bondad de ajuste satisfactorios y consistencia interna adecuadas.

Tabla 3

Análisis preliminar del segundo piloto $(N=192)$

\begin{tabular}{|c|c|c|c|c|c|c|c|}
\hline Ítems & M & DS & g1 & g2 & F1 & F2 & $\mathbf{h}^{2}$ \\
\hline Ítem1 & 2.72 & .48 & -1.39 & .85 & .50 & & .48 \\
\hline Ítem2 & 2.55 & .58 & -0.86 & -.26 & .56 & & .55 \\
\hline Ítem4 & 2.66 & .52 & -1.12 & .15 & .53 & & .46 \\
\hline Ítem9 & 2.54 & .59 & -0.90 & -.17 & .40 & & .28 \\
\hline Ítem10 & 1.95 & .59 & 0.01 & -.13 & .79 & & .67 \\
\hline Ítem12 & 2.55 & .70 & -1.24 & .16 & .90 & & .61 \\
\hline Ítem18 & 2.06 & .70 & -0.08 & -.92 & .37 & & .44 \\
\hline Ítem 20 & 2.43 & .62 & -0.59 & -.57 & .70 & & .39 \\
\hline Ítem 24 & 2.39 & .65 & -0.58 & -.63 & .38 & & .72 \\
\hline Ítem 25 & 2.59 & .60 & -1.19 & .37 & .52 & & .49 \\
\hline Ítem5 & 2.55 & .60 & -0.98 & -.03 & & .40 & .40 \\
\hline Ítem16 & 2.63 & .53 & -1.03 & .01 & & .39 & .50 \\
\hline Ítem17 & 2.57 & .66 & -1.25 & .31 & & .63 & .30 \\
\hline Ítem19 & 2.42 & .61 & -0.54 & -.61 & & .51 & .22 \\
\hline Ítem23 & 2.62 & .62 & -1.41 & .86 & & .22 & .44 \\
\hline F1 & & & & & 1.000 & & \\
\hline F2 & & & & & .68 & 1.000 & \\
\hline$\%$ varianza total & & & & & & 34.3 & \\
\hline Consistencia interna $(\omega)$ & & & & & .84 & .46 & \\
\hline
\end{tabular}

Nota $:$ M = Media; DS = Desviación Estándar; g1 = Asimetría; g2 = Curtosis, $\mathrm{h}^{2}$ : comunalidades.

En cuanto se refiere a los índices de bondad de ajuste para los tres modelos se obtuvieron a través de $\mathrm{SB}-X^{2} / g l \leq 2$ considerados los más adecuados (ver tabla 5); asimismo, el índice de ajuste comparativo $C F I \geq .85$ (Brown, 2006), TLI $>.90$ (Jöreskog \& Sörbom, 1986). SRMR $\leq .08$, RMSEA $\leq .08$ (Hu \& Bentler, 1999) y criterio de Akaike (Caballero, 2011), menor valor evidencia mayor parsimonia. Por lo tanto, se concluye que el M3 unifactorial es el modelo que mejor representa al constructo de estilo de afrontamiento al estrés en padres con hijos discapacitados. 
Tabla 4

Pesos factoriales, coeficiente omega y relación entre dimensiones de la Escala de Afrontamiento (Modelo 2 y 3 )

\begin{tabular}{|c|c|c|c|c|c|}
\hline \multirow{2}{*}{ Ítems } & \multicolumn{2}{|c|}{ Modelo 1} & \multicolumn{2}{|c|}{ Modelo 2} & \multirow{2}{*}{$\begin{array}{c}\text { Modelo3 } \\
\text { F1 } \\
\end{array}$} \\
\hline & $\mathrm{F} 1$ & F2 & $\mathrm{F} 1$ & $\mathrm{~F} 2$ & \\
\hline 1 & .480 & & .480 & & .480 \\
\hline 2 & .590 & & .590 & & .600 \\
\hline 4 & .430 & & .430 & & .430 \\
\hline 9 & .480 & & .480 & & .470 \\
\hline 10 & .750 & & .750 & & .750 \\
\hline 12 & .690 & & .690 & & .700 \\
\hline 18 & .430 & & .430 & & .430 \\
\hline 20 & .460 & & .450 & & .460 \\
\hline 24 & .400 & & .400 & & .400 \\
\hline 25 & .460 & & .460 & & .460 \\
\hline 5 & & .260 & & - & \\
\hline 16 & & .350 & & .340 & \\
\hline 17 & & .510 & & .420 & \\
\hline 19 & & .290 & & .380 & \\
\hline Consistencia interna $(\omega)$ & 0.79 & 0.37 & 0.79 & 0.33 & 0.79 \\
\hline $\mathrm{F} 1$ & 1 & & 1 & & \\
\hline F2 & 0.20 & & 0.24 & & \\
\hline
\end{tabular}

Nota: F1=Centrado en el problema; F2= Centrado en la emoción; $\omega=$ Omega

Tabla 5

Índices de bondad de ajuste de dos modelos de la Escala de afrontamiento

\begin{tabular}{lcccccccc}
\hline Modelo & SB- $\chi^{2}$ & gl & SB- $\chi^{2} /$ gl & CFI & TLI & SRMR & RMSEA & AIC \\
\hline M1: dos factores oblicuos (14 ítems) & 111.14 & 76 & 1.46 & .90 & .92 & .06 & .05 & 169.14 \\
M2: dos factores oblicuos (13 ítems) & 105.05 & 64 & 1.64 & .89 & .92 & .06 & .06 & 159.05 \\
M3: unifactorial (10 ítems) & 62.53 & 35 & 1.79 & .92 & .94 & .06 & .06 & 102.53 \\
\hline
\end{tabular}

Nota.: $\mathrm{SB}-\chi^{2}=$ corrección del chi cuadrado, gl=grado de libertad, $\mathrm{CFI}=$ índice de ajuste comparativo, $\mathrm{TLI}=$ índice Tacker Lewis, SRMR = raíz residual estandarizada cuadrática media, RMSEA= error cuadrático medio de aproximación; AIC $=$ Criterio de Información de Akaike.

\section{DISCUSIÓN}

Existen situaciones que son estresantes según la perspectiva de cada persona las cuales deben ser confrontadas por estrategias cognitivas y conductuales (Lazarus \& Folkman, 1986), los padres que tienen hijos con discapacidad se someten a 
mayores momentos de estrés (Cabezas, 2001; Badia, 2005; Sánchez, 2006) y enfrentan crisis en diferente áreas de su vida (Nuñez,2003), el INEI (2017) muestra que el $4.8 \%$ que presenta una situación de discapacidad se encuentra en la infancia hasta la adolescencia, indicando que se encuentran al cuidado de una persona adulta, Badia y Aguado (2000) señalan que el apoyo familiar es de suma importancia para el crecimiento de los sujetos con discapacidad, por lo expuesto vemos pertinente la creación y validación de una escala que mida el afrontamiento en padres que tienen hijos con discapacidad.

Exponemos los resultados obtenidos en cada uno de las fases del estudio del instrumento. La validez basada en el contenido fue realizada por el criterio de jueces, reside en aprobar o desaprobar uno o varios ítems de una prueba por diversos especialistas del tema, los puntajes pueden variar según el autor del instrumento (Escurra, 1988).de los 30 items establecido solo dos puntuaron por debajo del rango (V aiken $>.70)$.

En el estudio 2, como primer punto se realizó el AFE obteniendo varianza total de $34.3 \%$, estos resultados no se alejan de los obtenidos en los trabajos de muestra similar (Zorrilla, 2017).

En el AFC se obtuvo tres modelos distintos, el modelo 1 y en el modelo 2 (eliminado el ítem 4) los resultados se encuentran dentro de los rangos. El modelo 3 unidimensional - Centrado en el Problema, evidencia mejor índice de bondad de ajuste.

Los puntajes de las cargas factoriales en el AFC de los 3 modelos oscilan de .26 y .75 , de .34 - .75 y. .40 - .75, respectivamente, asimismo la correlación es .19 en el modelo 1 y .24 en el modelo 2.

Por lo tanto, el modelo 2, al parecer estaría presentando mejor ajuste en comparación al primer modelo, el Factor 2 - denominado Centrada en la emoción presentó una baja confiabilidad, ejecutándose así un tercer modelo, unidimensional (Centrado en el problema) obteniendo coeficientes de confiabilidad dentro del rango aceptable.

Investigaciones manifiestan que el afrontamiento centrado en el problema es el más utilizado por padres de hijos con discapacidad (Husni, 2018), al igual que el estudio de Albarracín, Jaimes y Rey (2014), los participantes de esta muestra cuentan con asesoramiento psicológico (orientación, talleres, pautas de crianza, evaluación, seguimiento, etc.).

Las limitaciones del estudio fue no utilizar un muestreo probabilístico, no considerar datos sociodemográficos, de igual modo se debe considerar que los resultados hallados son solo primeros indicios y solo debe aplicarse en la misma muestra. 


\section{REFERENCIAS}

Albarracín, Á., Rey, L., \& Jaime, M. (2014). Estrategias de afrontamiento y características sociodemográficas en padres de hijos con trastornos del espectro autista. Coping Strategies and Socio-demographic Characteristics in Parents of Children with Autistic Spectrum Disorders Stratégies de copin. Revista Virtual Universidad Católica del Norte, 42, 111- 126. Recuperado de http://revistavirtual.ucn.edu.co/ index.php/RevistaUCN/article/view/498/1034

Alosaimi, F., Hossam, S., Ayedh, K., Abdullah, I., Aljuaydi, K., Alotaibi, A. \& Alfarisb, E. (2018). Stress and coping among consultant physicians working in saudi arabia. Annals of Saudi Medicine, 38(3), 214-224. doi:http://dx.doi.org/10.5144/02564947.2018.214

Badia, M. (2005). Calidad de vida familiar: La familia como apoyo a la persona con parálisis cerebral. Psychosocial Intervention, 14(3), 325-341. Recuperado de: http:// www.redalyc.org/pdf/1798/179817547006.pdf

Badia, M. \& Aguado, A. (2000). Escala de Estrés y Afrontamiento para familias con hijos afectados de Parálisis Cerebral. (Artículo Inédito).

Bandalos, D. \& Finney, S. (2010). Factor Analysis: Exploratory and Confirmatory. En G. R. Hancock y R. O. Mueller (Eds.), Reviewer's guide to quantitative methods. Routledge: New York

Barraza, C. (2018). Propiedades psicométricas del Cuestionario BriefCope28 en población española expuesta a eventos altamente estresante. http://coloquioenfermeria2018. sld.cu/index.php/coloquio/2018/paper/view/616/373

Barquín-Cuervo, R., Medina-Gómez, Ma B. y Pérez de Albéniz-Garrote, G. (2018). El uso de estrategias de afrontamiento del estrés en personas con discapacidad intelectual. Psychosocial Intervention, 27, 89-94. https://doi.org/10.5093/pi2018a7

Beaducel, A. \& Herzberg, P. Y. (2006). On the performance of maximum likelihood versus means and variance adjusted weighted least squares estimation in CFA. Structural Equation Modeling, 13, 186-203. doi: 10.1207/s15328007sem1302_2

Benson, P. R. (2014). Coping and Psychological Adjustment Among Mothers of Children with ASD: An Accelerated Longitudinal Study. Journal of Autism and Developmental Disorders, 44(8), 1793-1807. Recuperado de doi:10.1007/s10803-014-2079-9

Brown, T. A. (2006). Confirmatory factor analysis for applied research. New York: Guilford Press.

Caballero, F. (2011). Selección de modelos mediante criterio de información en análisis factorial. Aspectos teóricos y computacionales (tesis doctoral). Universidad de Granados: España. Recuperado de: http://digibug.ugr.es/bitstream/handle/10481/19 428/199648? sequence $=1$

Cabezas, M. (2001). Los padres del niño con autismo: Una guía de intervención. Revista Electrónica "Actualidades Investigativas en Educación”, 1 (2). Recuperado de: https://www.redalyc.org/pdf/447/44710202.pdf 
Campos-Arias, A. \& Oviedo H. (2008). Propiedades psicométricas de una Escala: Consistencia interna. Revista de Salud Pública, 10 (5), 831-839. Recuperado de http:// www.redalyc.org/pdf/422/42210515.pdf

Cano, F., Rodriguez, L. \& Garcia, J. (2007). Adaptación española del Inventario de Estrategias de Afrontamiento. Actas Españolas de Psiquiatría, 35(1), 29-39https://idus. us.es/xmlui/bitstream/handle/11441/56854/Preprint_2007_Adaptaci\%C3\%B3n\%20 espa $\%$ C3\%B1ola $\% 20 \mathrm{del} \% 20$ Inventario $\% 20 \mathrm{de} \% 20$ Estrategias $\% 20 \mathrm{de} \% 20$ Afrontamiento.pdf?sequence $=1 \&$ is Allowed $=\mathrm{y}$

Cappe, E., Wolf, M., Bobet, R. \& Adrien, J. (2011) Quality of life: a key variable to consider in the evaluation of adjustment in parents of children with autism spectrum disorders and in the development of relevant support and assistance programmes. Qual Life Res Int J Qual Life Asp Treat Care Rehabil, 20(8), 1279-1294. Recuperado de https:// doi.org/10.1007/s11136-011-9861-3

Carretero-Dios, H., \& Pérez, C. (2005). Normas para el desarrollo y revisión de estudios instrumentales. International Journal of Clinical and Health Psychology, 5 (3), 521551. Recuperado de http://www.redalyc.org/articulo.oa?id=33705307

Carter, A., Martínez-Pedraza, F.\& Gray, S. (2009). Stability and individual change in depressive symptoms among mothers raising young children with ASD: maternal and child correlates. Journal of Clinical Psychology, 65(12), 1270-1280. Recuperado de doi:10.1002/jclp.20634

Carver, C., Scheier, M., \& Weintraub, J. K. (1989). Assessing Coping Strategies: A theoretically based approach. Journal of Personality and Social Psychology.

Carver, C. S. (1997). You want to measure coping but your protocol's too long: Consider the Brief COPE. International Journal of Behavioral Medicine, 4(1), 92-100.

Cassaretto M. \& Perez-Aranibar C. (2016). Afrontamiento al Estrés: Adaptación del Cuestionario COPE en Universitario de Lima. Revista Iberoamericana de Diagnóstico y Evaluación Psicológica, 42(2), 95-109. Recuperado de http://www. aidep.org/sites/default/files/articles/R42/Art9.pdf

Casu, G., Zaia, V., Fernandes Martins, M. d. C., Parente Barbosa, C., \& Gremigni, P. (2019). A dyadic mediation study on social support, coping, and stress among couples starting fertility treatment. Journal of Family Psychology, 33(3), 315-326. Recuperado de https://www.ncbi.nlm.nih.gov/pubmed/30730186

Charles, V., Santoro, M., Edwards, L., Kang, J. \& Cronan, T. (2016). Assessing the structure of the ways of coping questionnaire in fibromyalgia patients using common factor analytic approaches. Pain Research \& Management,2016. Recuperado de :doi:http:// dx.doi.org/10.1155/2016/7297826

Charter, R. (2003). A breakdown of reliability coefficients by test type and reliability method, and the clinical implications of low reliability. Journal of General Psychology, 130 (3), 290-304.

Chorot, P., y Sandín, B. (1987). Escala de Estrategias de Coping (EEC). Madrid: Universidad Nacional de Educación a Distancia. (Policopiado). 
Connor-Smith, J. \& Compas, B. (2004). Coping as a moderator of relation between reactivity to interpersonal stress, health status and internalizing problems. Cognitive and Therapy Research, 28 (3), 347-368.

Craşovan, D., \& Sava, F. (2013). Translation, adaptation, and validation on romanian population of COPE Questionnaire for Coping Mechanisms Analysis. Cognition, Brain \& Behavior. An Interdisciplinary Journal, 17(1), 61-76.

Crespo, M., \& Cruzado, J. (1997). La evaluación del afrontamiento: Adaptación Española del Cuestionario COPE con una muestra de estudiantes universitarios. Análisis y Modificación de Conducta, 23(92), 797-830

Dabrowska, A. \& Pisula, E. (2010) estrés de los padres y estilos en las madres y los padres de niños en edad preescolar con autismo y síndrome de Down afrontamiento. J Intellect Disabil Res, 54 (3): 266-280.Recuperado de https: // doi.org/10.1111 /j.1365-2788.2010.01258.x

Dardas, L. \& Ahmad, M. (2015). Coping strategies as mediators and moderators between stress and quality of life among parents of children with autistic disorder. Stress Health J Int Soc Invest Stress, 31(1), 5-12. https://doi.org/10.1002/smi.2513

Díaz, C., Abal, Y., Climent, J. A., Ortega, E., López-López, M., \& Casado, J. (2016). Pérdida y afrontamiento en desempleados: Adaptación del Inventario Texas Revisado de Duelo a la situación de pérdida de empleo [Loss and coping in unemployed: Adaptation of the Texas Revised Inventory of Grief to the situation of loss of employment]. Revista Iberoamericana de Diagnóstico y Evaluación Psicologica, 42(2), 111-124.

Escurra, L. (1). Cuantificación de la validez de contenido por criterio de jueces. Revista De Psicología, 6(1-2), 103-111. Recuperado a partir de http://revistas.pucp.edu.pe/ index.php/psicologia/article/view/4555

Fantova, F.(2000). Trabajar con las familias de las personas con discapacidad. Siglo Cero, 31(6), 192.

Folkman, S. \& Moskowitz, J. (2004). Coping: pitfalls and promise. Annual Review of Psychology, 55, 745-774.

Frydenberg, E. y Lewis, R. (1999). Things donít better just because youíre older: A case for facilitating reflection. British Journal of Educational Psychology, 69; 81-94.

Gana, K. \& Broc, G. (2019). Structural Equation Modeling with Lavaan. New York: Wiley

George, D. \& Mallery, P. (2003). Using SPSS for Windows step by step: a simple guide and reference, MA: Allyn \& Bacon. Recuperado de https://wps.ablongman.com/ wps/media/objects/385/394732/george4answers.pdf

González, M. \& Landero, R. (2007). Cuestionario de afrontamiento del estrés (CAE): validación en una muestra mexicana. Revista de Psicopatología y Psicología Clinica, 12(3). Recuperado de:https://doi.org/10.5944/rppc.vol.12.num.3.2007.4044

Guarino, L., Sojo, V. \& Bethelmy, L. (2007). Adaptación y validación preliminar de la versión hispana del cuestionario de estilos de afrontamiento (coping style questionnaire, CSQ). Psicologia Conductual, 15 (2), 173-189. Recuperado de https://www.researchgate.net/ profile/Victor_Sojo/publication/220006627_Adaptacion_y_Validacion_Preliminar_ 
de_la_Version_Hispana_del_Cuestionario_de_Estilos_de_Afrontamiento_Coping_ Style_Questionnaire_CSQ/links/0c96051f0760 bc21a8000000.pdf

Hernández, R., Fernández, C. y Baptista, P. (2014). Metodología de la investigación (6 ${ }^{\mathrm{a}}$ ed.). México DF: McGraw Hill.

Herrera P., M. S., Fernández L., M. B., \& Barros L., L. C. (2018). Estrategias de afrontamiento en relación con los eventos estresantes que ocurren al envejecer. Ansiedad y Estrés, 24(1), 47-52. http://dx.doi.org.ezproxybib.pucp.edu.pe:2048/10.1016/j. anyes.2017.10.008

Hooper, D., Coughlan, J. y Mullen, MR (2008). Modelado de ecuaciones estructurales: pautas para determinar el ajuste del modelo. The Electronic Journal of Business Research Methods, 6, 53-60.

Hu, L. T. y Bentler, P. M. (1999). Cutoff criteria for fit indexes in covariance structure analysis: Conventional criteria versus new alternatives. Structural Equation Modeling, 6, 1-55. https://doi.org/10.1080/10705519909540118

Husni, M. (2018). Stress Levels and Coping Strategies among Parents of Children with Autism and Down Syndrome: The Effect of Demographic Variables on Levels of Stress. Child Care in Practice,25, 146-156. Recuperado de: https://www.tandfonline. com/doi/full/10.1080/13575279.2018.1446907

Ingledew, D. K., Hardy, L., Cooper, C.L., \& Jemal, H. (2011). Health behaviors reported as coping strategies: A factor analytical study. British Journal of Health Psychology, 1(3), 263-281. Recuperado de https://www.researchgate.net/publication/230206972 Health_behaviours_reported_as_coping_strategies_A_factor_analytical_study

Instituto Nacional de Estadística e Informática, INEI. (2017). Perfil Sociodemográfico Informe Nacional. Recuperado de: https:/www.inei.gob.pe/media/MenuRecursivo/ publicaciones_digitales/Est/Lib1136/libro.pdf

Izquierdo, G., García, G., García, C., Delgado, M., Jiménez, G., \& Vázquez, V. (2008). Propiedades psicométricas de la Escala de Autoeficacia para el Afrontamiento del Estrés (EAEAE). Psicothema, 20(1), 155-165.

Ji, B., Zhao, I., Turner, C., Sun, M., Yi, R. \& Tang, S. (2014). Predictors of health-related quality of life in Chinese caregivers of children with autism spectrum disorders: a cross-sectional study. Arch Psychiatr Nurs 28(5),327-332. https://doi.org/10.1016/j. apnu.2014.06.001

Jöreskog, K. \& Sörbom, D. (1986). LISREL VI: Analysis of Linear Structural Relationships by Maximum Likelihood and Least Squares Methods. Mooresville, IN: Scientific Software, Inc.

Kallasmaa, T., \& Pulver, A. (2000). The structure and properties of the Estonian COPE inventoy. Personality and Individual Differences, 29, 881-894.

Kline, R. (2010). Principles and practice of structural equation modeling. New York: Guilford Press. 
Lai, W., Goh, T., Oei, T., \& Sung, M. (2015). Coping and Well-Being in Parents of Children with Autism Spectrum Disorders (ASD). Journal of Autism and Developmental Disorders, 45(8), 2582-2593. doi:10.1007/s10803-015-2430-9

Laskowska, A. (2018). Quality of life in cancer as a function of temperament, coping and posttraumatic stress disorder. Current Psychology, 1-9. doi:http://dx.doi.org/10.1007/ s12144-018-0008-3

Lazarus, R. S. \& Folkman, S. (1984). Stress, appraisal and coping. Nueva York: Springer Publishing Company.

Lazarus, R. S. \& Folkman, S. (1986). Estrés y procesos cognitivos. Barcelona: Martínez Roca.

Livneh, H., y Wilson, LM (2003). Estrategias de afrontamiento como predictores y mediadores de las variables relacionadas con la discapacidad y la adaptación psicosocial: una investigación exploratoria. Boletín de consejería de rehabilitación, 46 (4), 194-208. https://doi.org/10.1177/003435520304600401

Londoño, N.,Henao López, G., Puerta, I.,Posada, S., Arango, D., \& Aguirre Acevedo, D. (2006). Propiedades psicométricas y validación de la escala de estrategias de coping modificada (EEC-M) en una muestra colombiana. Universitas Psychologica, 5 (2), 327-349. Recuperado de https://www.redalyc.org/articulo.oa?id=64750210

López, X. (1999).Un modelo de Trabajo con padres de niños discapacitados en Base a la Teoría de Duelo. PSYKHE (8)2, 111-117.

Lorenzo-Seva, U. (1999). Promin: A method for oblique factor rotation. Multivariate Behavioral Research, 34(3), 347-365. Recuperado de https://www.tandfonline.com/ doi/abs/10.1207/S15327906MBR3403_3

Lorenzo-Seva, U., \& Ferrando, P. J. (2015). Factor 10.3. Spain: Rovira I Virgili University.

Lostaunau, V., Torrejón, C., \& Cassaretto, M. (2017). Estrés, afrontamiento y calidad de vida relacionada a la salud en mujeres con cáncer de mama. Actualidades en Psicología, 31(122), 75-90. http://dx.doi.org.ezproxybib.pucp.edu.pe:2048/10.15517/ ap.v31i122.25345

Luque, B., Yáñez, V., Tabernero, C. \& Cuadrado, E. (2017) The role of coping strategies and self-efcacy as predictors of life satisfaction in a sample of parents of children with autism spectrum disorder. Psicothema, 29(1), 55-60.

Lyne, K., \& Roger, D. (2000). A psychometric re-assessment of the COPE questionnaire. Personality and Individual Differences, 29, 321-335.

Mardia, K.V. (1970). Measures of multivariate skewness and kurtosis with applications. Biometrika, 57, 519-530.

Mardia, K.V. (1974). Applications of some measures of multivariate skewness and kurtosis in testing normality and robustness studies. Sankhya, B36, 115-128.

Mate, A., Andreu, J. \& Peña, M. (20169. Propiedades psicométricas de la versión española del "Inventario breve de afrontamiento (COPE-28) en una muestra de adolescentes". Behavioral Psychology, 24 (2), 305-318 .Recuperado de https://www.researchgate. 
net/profile/Jose_Andreu2/publication/309482569_Psychometric_properties_of_ the_Spanish_version_of_the_Brief_COPE_Inventory_COPE-28_in_a_sample of_teenagers/links/5857050708ae77ec3709438f/Psychometric-properties-of-theSpanish-version-of-the-Brief-COPE-Inventory-COPE-28-in-a-sample-of-teenagers. pdf

Marsh, H. W. y Tai Hau, k. (1996). Assessing Goodness of Fit: Is Parsimony Always Desirable? The Journal of Experimental Education, 64 (4), 364-390. Recuperado de http://www.jstor.org/stable/20152499

Mayo, M., Real, J., Taboada, E., Iglesias-Souto, P. \& Dosil, A. (2012). Análisis de las propiedades psicométricas del Cuestionario de Formas de Afrontamiento de Acontecimientos Estresantes (C.E.A.), aplicado a padres de niños con discapacidad visual. Anales de la Psicología, 28(1), 83-88. Recuperado de http://www.redalyc.org/pdf/167/16723161010.pdf

McDonald, R. P. (1999). Test theory: A unified treatment. Mahwah: Lawrence Erlbaum Associates, Inc

Miyazaki, Y., Bodenhorn, N., Zalaquett, C., \& KokMun, N. (2008). Factorial structure of Brief COPE for international students attending U.S. colleges. College Student Journal, 42(3), 795- 806.

Morán, C., Landero, R. \& González, M.T. (2010). COPE-28: un análisis psicométrico de la versión en español del Brief COPE. Universitas Psychologica, 9 (2), 543-552. Recuperado de http://www.scielo.org.co/pdf/rups/v9n2/v9n2a20.pdf

Muller, L. \& Spitz, E. (2003). Evaluation multi-dimensionelle du coping: Validation du Brief COPE sur une population francaise. L'Encephale, XXIX, 507-518.

Muñiz, J. (2005). Classical test models. En B. S. Everitt and D. C. Howell (Eds.), Encyclopedia of Statistics in Behavioral Science. Chichester: John Wiley and Sons. (pp. 278-282).

Muthén, B. y Kaplan D. (1985). A comparison of some methodologies for the factor analysis of non-normal Likert variables. British Journal of Math-ematical and Statistical Psychology, 38, 171-189. Recuperado de: http://www.statmodel.com

Nava, C., Ollua, P., Vega, C. \& Soria R. (2010). Inventario de Estrategias de Afrontamiento: una replicación. Psicología y Salud 20 (2), 213-220. Recuperado de https://www. uv.mx/psicysalud/psicysalud-20-2/20-2/Carlos-Nava-Quiroz.pdf

Núñez, B. (2003). La familia con un hijo con discapacidad: sus conflictos vinculares. Archivos argentinos de pediatría, 101(2). Recuperado de https:/www.sap.org.ar/ docs/publicaciones/archivosarg/2003/133.pdf

Padilla, J., Gómez, J., Hidalgo, M. \& Muñiz, J.(2007). Esquema conceptual y procedimientos para analizar la validez de las consecuencias del uso de los tests. Psicothema, 19(1), 173-178. Recuperado de: http://www.psicothema.com/psicothema.asp?id=3345

Parrello, S. \& Caruso, E. (2007). El nacimiento de un hijo con minusvalía: ruptura de cánones y posibilidad de narrarse. Revista Electrónica de Psicología Iztacala 
10 (1). Recuperado de: http://www.revistas.unam.mx/index.php/repi/article/ view/18980/18006

Perczek, R., Carver, C. S., Price, A. A., \& Pozo-Kaderman, C. (2000). Coping, mood, and aspects of personality in Spanish translation and evidence of convergence with English versions. Journal of Personality Assessment, 74(1), 63- 87. doi: 10.1207/ S15327752JPA740105

Pozo, P., Sarriá, E. \& Méndez, L. (2006). Estrés en madres de personas con trastorno del espectro autista. Psicothema, 18(1), 342-347. Recuperado de: http://www.psicothema. com/pdf/3220.pdf

Quezada-Berumen, L., Moral de la Rubia, J., Ibarra-González, L., \& González-Ramírez, M. (2018). Estudio de validación del Cuestionario de Afrontamiento del Estrés en personas trans de México. Revista de Psicopatología y Psicología Clínica, 23(2), 121-134. doi:https://doi.org/10.5944/rppc.vol.23.num.2.2018.19367

Quiroz, C., Vega Valero, C., \& Soria Trujano, R. (2010). Escala de Modos de Afrontamiento: consideraciones teóricas y metodológicas. Universitas Psychologica, 9(1), 139-147. Recuperado a partir de https://revistas.javeriana.edu.co/index.php/revPsycho/article/ view/274

Reich, M., Costa-Ball, C. \& Remor, Eduardo. (2016). Estudio de las propiedades psicométricas del Brief COPE para una muestra de mujeres uruguayas. Avances en Psicología Latinoamericana, 34(3), 615-636. https://dx.doi.org/10.12804/ ap134.3.2016.13

Renati. (2018). Registro Nacional de trabajos de investigación. Recuperado de http:// renati.sunedu.gob.pe/simple-search?query=afrontamiento

Rexrode, K. R. (2005). The ways of coping questionnaire: A reliability generalization study (Order No. 3178828). Available from ProQuest Central. (305447180). Retrieved from https://search.proquest.com/docview/305447180?accountid=37408

Rieger, S. M. (2016). Coping strategy assessment in traumatic brain injured patients: Reliability, validity, and factor structure of the ways of coping questionnairetraumatic brain injury (WCQ-TBI) (Order No. 10119450). Available from ProQuest Central. (1805577780). Retrieved from https://search.proquest.com/docview/1805577 780 ? accountid $=37408$

Rodríguez-Díaz, F., Estrada-Pineda, C., Rodríguez-Franco, L., \& Bringas-Molleda, C. (2014). Adaptación del Inventario de Estrategias de Afrontamiento (CSI) a la Población Penitenciaria de Mexico. Psicologia: Reflexão e Crítica, 27 (3), 415-423. Recuperado de https://www.redalyc.org/articulo.oa?id=18831844001

Rodríguez- Marín, J., Terol M., López - Roig, S. \& Pastor, M. (1992). Evaluación del afrontamiento del estrés: Propiedades psicométricas del cuestionario de formas de afrontamiento en acontecimientos estresantes. Journal of Health Psychology, 4(2), 59-83. Recuperado de https://www.researchgate.net/publication/282715137 Evaluacion_del_afrontamiento_del_estres_Propiedades_psicometricas_del_ cuestionario_de_formas_de_afrontamiento_de_a contecimientos_estresantes 
Roger, D., Jarvis, G. y Najarian, B. (1993). Detachment and coping: The construction and validation of a new scale for measuring coping strategies. Personality and Individual Differences, 15 (6), 619-626

Roger, D. (1995). The mechanics of stress: a model for the relationship between stress, health and personality. Comunicación presentada en la 5ta. Conferencia Internacional en Manejo del Estrés, Noordwijkerhout, Holanda.

Sánchez, P. (2006). Discapacidad, familia y logro escolar. Revista Iberoamericana de Educación, 40 (2). Recuperado de: http://riberdis.cedd.net/handle/11181/4402

Sandín, B. (2002). El estrés: un análisis basado en el papel de los factores sociales. International Journal of Clinical and Health Psychology, 3(1), 141-157. Recuperado de https://www.redalyc.org/pdf/337/33730109.pdf

Sandín, B. \& Chorot, P. (2002). Cuestionario de Afrontamiento del Estrés (CAE): Desarrollo y Validación Preliminar. Universidad Nacional de Educación a Distancia. Recuperado de: http://www.aepcp.net/arc/03.2003(1).Sandin-Chorot.pdf

Satorra, P., \& Bentler, P. (2001). A scaled difference chi-square test statistic for moment structure analysis. Psychometrika, 66(4), 507-514.

Sawang, S., Oei, T. P. S., Goh, Y. W., Mansoer, W., Markhum, E., \& Ranawake, D. (2010). Confirmatory factor analysis of the way of coping checklist-revised (WCCL-R) in the asian context. Applied Psychology, 59(2), 202-219. doi:http://dx.doi.org/10.1111/ j.1464-0597.2009.00378.x context. Applied Psychology, 59(2), 202-219. doi:http:// dx.doi.org/10.1111/j.1464-

Scherer, R. F., \& Brodzinski, J. D. (1990). AN ANALYSIS OF THE WAYS OF COPING QUESTIONNAIRE research instrument. Management Communication Quarterly: $\mathrm{McQ}$, 3(3), 401. Recuperado de https://search.proquest.com/docview/232862935?ac countid $=37408$

Sürücü, H. A., Besen, D. B., Duman, M., \& Erbil, E. Y. (2018). Coping with stress among pregnant women with gestational diabetes mellitus. Journal of Caring Sciences, 7(1), 9-15. doi:http://dx.doi.org/10.15171/jcs.2018.002

Tabachnick, B. \& Fidell, L. (2007), Using Multivariate Statistics (5th ed.). New York: Allyn and Bacon.

Tobin, D. L., Holroyd, K. A., \& Reynolds, R. (1982). The assessment of coping: Psvchometric development of the Coping Strategies Inventory. Paper presented at the meeting for the Advancement of Behavior Therapy, Los Angeles.

Tobin, D. L., Holroyd, K. A., \& Reynolds, R. (1983). The measurement of coping in response to stress: The Coping Strategies Inventory. Paper presented at the meeting of the Society of Behavioral Medicine, Baltimore

Tobin, D., Holroyd K., Reynolds, R. \& Kigal, J. (1989).The hierarchical factor structure of the Coping Strategies Inventory. Cognit Ther Res, 13(4), 343-361

Vargas-Manzanares, S., Herrera -Olaya, G., Rodríguez -García, L., \& Sepúlveda -Carrillo, G. (2011). Confiabilidad del cuestionario Brief COPE inventory en versión en español para evaluar estrategias de afrontamiento en pacientes con cáncer de seno. 
Investigación En Enfermería: Imagen Y Desarrollo, 12(1), 7-24. Recuperado a partir de https://revistas.javeriana.edu.co/index.php/imagenydesarrollo/article/view/1622

Violanti, J. M., Ma, C. C., Mnatsakanova, A., Fekedulegn, D., Hartley, T. A., Gu, J. K., \& Andrew, M. E. (2018). Associations between police work stressors and posttraumatic stress disorder symptoms: Examining the moderating effects of coping. Journal of Police and Criminal Psychology, 33(3), 271-282. doi:http://dx.doi.org/10.1007/ s11896-018-9276-y

Wang, J., Hu, Y., Wang, Y., Qin, X., Xia, W., Sun, C. \& Wang, J. (2013). Parenting stress in Chinese mothers of children with autism spectrum disorders. Social Psychiatry and Psychiatric Epidemiology, 48(4), 575-582. doi:10.1007/s00127-012-0569-7

Wei-Po Chou, Cheng-Fang, Y., \& Tai-Ling, L. (2018). Predicting effects of psychological Inflexibility/Experiential avoidance and stress coping strategies for internet addiction, significant depression, and suicidality in college students: A prospective study. International Journal of Environmental Research and Public Health, 15(4), 788. doi:http://dx.doi.org/10.3390/ijerph15040788

Whitehead, B. R., \& Bergeman, C. S. (2019). Daily religious coping buffers the stressaffect relationship and benefits overall metabolic health in older adults. Psychology of Religion and Spirituality. Advance online publication. http://dx.doi.org.ezproxybib. pucp.edu.pe:2048/10.1037/rel0000251

Zorrilla, E. (2017). Adaptación del cuestionario de afrontamiento al estrés en padres de familia de niños con discapacidad del C.E.B.E Manuel Duato. (Tesis Pregrado). Universidad César Vallejo. Perú. 


\section{Anexo 1}

Escala de Afrontamiento en Padres de Hijos con Discapacidad

Centrado en el problema

Autores: Araseli Yaquelin Tanta-Luyo, Marisela Quispe Fernández, AntonioSerpa Barrientos, Daniella Elizabeth Ardiles Guevara

Edad:

Sexo:

Grado de instrucción:

Discapacidad del menor:

Presentamos una lista de afirmaciones, marque con una (x) dentro del recuadro, la que usted se identifica frecuentemente.

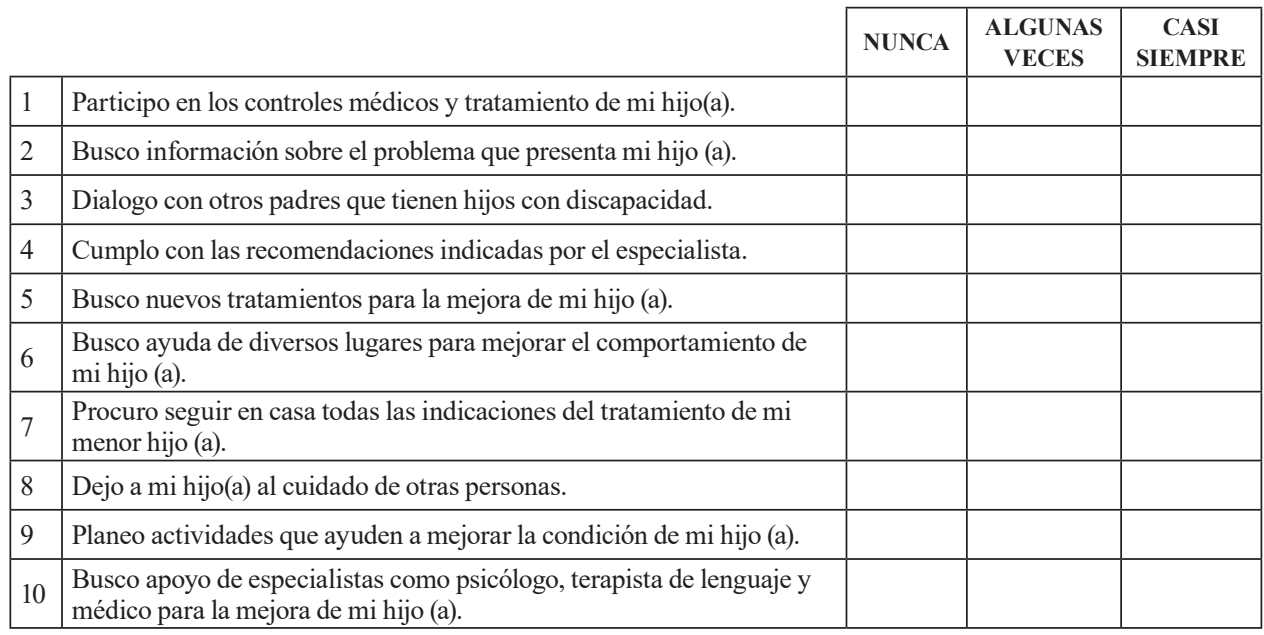


